\title{
Quantum key distribution based on a Sagnac loop interferometer and polarization-insensitive phase modulators
}

\author{
Bing Qi, Lei-Lei Huang, Hoi-Kwong Lo, Li Qian \\ Center for Quantum Information and Quantum Control \\ Dep. of Physics and Dep. of Electrical and Computer Engineering \\ University of Toronto, Toronto, Canada M5S 3G4 \\ Email: bqi@physics.utoronto.ca, leilei.huang@utoronto.ca \\ hklo@comm.utoronto.ca, l.qian@utoronto.ca
}

\begin{abstract}
We present a design for a quantum key distribution (QKD) system in a Sagnac loop configuration, employing a novel phase modulation scheme based on frequency shift, and demonstrate stable BB84 QKD operation with high interference visibility and low quantum bit error rate (QBER). The phase modulation is achieved by sending two light pulses with a fixed time delay (or a fixed optical path delay) through a frequency shift element and by modulating the amount of frequency shift. The relative phase between two light pulses upon leaving the frequency-shift element is determined by both the time delay (or the optical path delay) and the frequency shift, and can therefore be controlled by varying the amount of frequency shift. To demonstrate its operation, we used an acousto-optic modulator (AOM) as the frequency-shift element, and vary the driving frequency of the AOM to encode phase information. The interference visibility for a $40 \mathrm{~km}$ and a $10 \mathrm{~km}$ fiber loop is 96\% and 99\%, respectively, at single photon level. We ran BB84 protocol in a 40-km Sagnac loop setup continuously for one hour and the measured QBER remained within the $2 \% \sim 5 \%$ range. $A$ further advantage of our scheme is that both phase and amplitude modulation can be achieved simultaneously by frequency and amplitude modulation of the AOM's driving signal, allowing our QKD system the capability of implementing other protocols, such as the decoy-state QKD and the continuous-variable QKD. We also briefly discuss a new type of Eavesdropping strategy ("phaseremapping" attack) in bidirectional QKD system.
\end{abstract}

\section{INTRODUCTION}

One important practical application of quantum information is quantum key distribution (QKD), whose unconditional security is based on the fundamental law of quantum mechanics [1-6]. In principle, any eavesdropping attempt by a third party (Eve) will unavoidably introduce quantum bit errors, so, it's possible for the legitimate users (Alice and Bob) to upper bound the amount of information acquired by the eavesdropper from some system parameters and the measured quantum bit error rate (QBER). Alice and Bob can then distill out a final secure key by performing error correction and privacy amplification. Because Alice and Bob can't distinguish the intrinsic QBER due to imperfections in a practical QKD system from the one induced by Eve, to guarantee the unconditional security, they have to assume all errors originate from eavesdropping. Obviously, the QKD system with higher intrinsic QBER will yield a lower secure key rate. As the QBER reaches some threshold, QKD is not unconditional secure anymore.

In a practical phase-coding QKD system, Alice and Bob achieve phase encoding/decoding with phase modulators (PM) and Mach Zehnder interferometers (MZI) [7-8]. However, there are a few practical difficulties if QKD is to be implemented over long distance through fiber: namely, phase and polarization instabilities. In this case, the intrinsic QBER induced by the imperfect interference can be described as [2]

$$
Q B E R=(1-V) / 2
$$

where $\mathrm{V}$ is the interference visibility.

Although promising progresses have been achieved by using active feedback control to stabilize the interferometer [8], the "plug \& play" auto-compensating QKD structure employing a Faraday mirror has demonstrated higher performance in practice [9]. Like the "plug \& play" system, the Sagnac loop also offers phase stability and polarization stability, as the two interfering signals travel through the same path, but its structure is in principle much simpler than the "plug \& play" scheme [10-13]. However, all reported Sagnac QKD systems employed polarization-sensitive phase modulators, requiring complicated polarization controls, which makes this scheme unattractive. For example, four polarization controllers were employed in [12], and the interference visibility for a $5 \mathrm{~km}$ fiber loop was only $87 \%$.

In this paper, we present a design for an AOM-based polarization-insensitive phase modulation scheme together with a Sagnac QKD system, and demonstrate stable QKD operation over one hour without feedback control. Although this system is designed for the BB84 protocol [1], with a few straightforward modifications, it can also be adapted to implement other protocols, such as the decoy state QKD [14$20]$ and the continuous variable QKD [21]. 


\section{AOM-BASED PHASE MODULATOR AND BIDIRECTIONAL SAGNAC QKD SYSTEM}

As two light pulses with a fixed time delay pass though a frequency shift element, a relative phase shift is introduced between the two pulses, which is determined by the amount of frequency shift. So, by modulating the frequency shift, phase modulation can be achieved. This is the basics principle of our phase modulation scheme.

Fig. 1a shows the basic structure of this novel phase modulator, which consists of an acousto-optic modulator (AOM), followed by a fiber with length $L$. For the first-order diffracted light, the AOM will introduce a frequency shift equal to its driving frequency $f$ (due to the Doppler effect). The phase of the diffracted light is also shifted by an amount of $\phi(t)$, which is the phase of acoustic wave at the time of diffraction [22]. Assuming two light pulses, $S_{1}$ and $S_{2}$, are in phase and are sent to the phase modulator at the same time from opposite directions as shown in Fig. 1a. They will reach the AOM at different times with a time difference $t_{2}-t_{1}=n L / C$. Here $n$ is effective index of fiber and $C$ is the speed of light in vacuum. The phase difference between $S_{1}$ and $S_{2}$ after they go through the phase modulator will be

$$
\Delta \phi=\phi\left(t_{2}\right)-\phi\left(t_{1}\right)=2 \pi f\left(t_{2}-t_{1}\right)=2 \pi n L f / C
$$

Fig. 1. a) A phase modulator with one frequency up-shifter; b) A phase modulator with a pair of frequency shifters. +: up-shifting AOM, -: downshifting AOM, L: fiber with length L, D: AOM driver

By modulating AOM's driving frequency $f$, the relative phase between $S_{1}$ and $S_{2}$ can be modulated. We remark that the frequency of light will be up-shifted by this phase modulator by an amount $f$. To remove this "side-effect", we can add another frequency down shifter at the other end of the fiber, as shown in Fig.1b. In Fig.1b, the two AOMs, which are driven by the same driver, will shift the frequency of light by the same amount but with different signs. So the net frequency shift will be zero. Since a down-shift AOM will shift the phase of the diffracted light by $-\phi(t)$, the resulting phase difference between $S_{2}$ and $S_{1}$ after they go through the phase modulator will be

$$
\Delta \phi=\phi_{S_{2}}-\phi_{S_{1}}=4 \pi n L f / C
$$

Compared with the $\mathrm{LiNbO}_{3}$ waveguide-based phase modulator, the AOM-based phase modulator we proposed can be designed to be insensitive to the polarization state of the input light. This could dramatically simplify the design of many QKD systems. As shown in (2) and (3), the phase delay is determined by the acoustic frequency $f$, which can be controlled very precisely $(1 \mathrm{ppm}$ frequency resolution is quite common). This implies that a high resolution phase modulation can be achieved with our design. As a comparison, the resolution of $\mathrm{LiNbO}_{3}$ phase modulator is in the order of $1 \%$.

We have proposed a Sagnac QKD system employing this novel phase modulator, as shown in Fig.2. To realize the BB84 protocol, Alice randomly encodes the relative phase between the clockwise and counterclockwise light pulses with the AOM-based phase modulator PM1, while Bob randomly chooses his measurement basis with phase modulator PM2. In Alice's side, two classical photo detectors (PD1, PD2) and two wavelength filters (F1, F2) are introduced to counter Trojan horse attack. These photo detectors could also be used for synchronization purpose.

We remark the transmittance of AOM can also be modulated by modulating the amplitude of its driving signal, so, the same device can function as an amplitude modulator as well as a phase modulator. (We remark that the newly developed decoystate QKD protocol [14-20], which improves the secure key generation rate of practical QKD system dramatically, can be easily realized in our proposed setup. In decoy-state QKD, Alice randomly adds decoy pulses, which are used for testing the communication channel, into the signal pulses for key distribution. The decoy pulses are identical to the signal pulses except for the average photon number. In the first experimental demonstration of the decoy QKD [20], we added an external AOM into a "plug \& play" system to randomly modulate the amplitude of each laser pulse according to a random profile. Because the phase modulator we proposed can also function as an amplitude modulator, our proposed QKD system can implement decoy state protocol easily: Alice can achieve phase modulation by modulating the frequency of the AOM driving signal, at the same time, she can also modulate the intensity of each pulse by modulating the amplitude of the driving signal.)

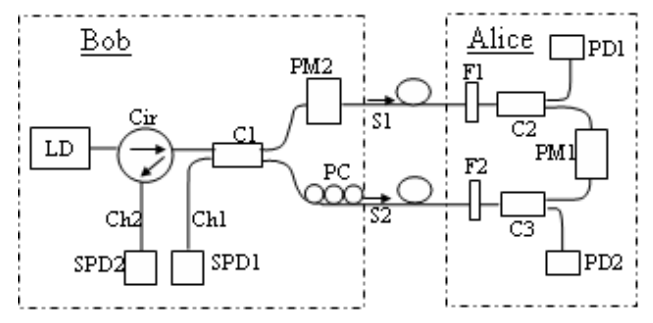

Fig. 2. Proposed QKD system: LD-pulsed laser diode; Cir-circulator; C-2x2 coupler; PC-polarization controller; F1,F2-optical filter; PD1, PD2classical photo detector; PM1, PM2- AOM-based phase modulator (as shown in Fig.1b); SPD1, SPD2-Single Photon detector

We remark, by introducing asymmetrical attenuation in the fiber loop (for example, fiber isolators) and replacing the SPDs with homodyne detector, continuous variable QKD [21] could be implemented with the proposed system: here, Alice can randomly modulates the amplitude quadrature and phase quadrature with PM1, while Bob chooses which quadrature to measure with PM2. 


\section{EXPERIMENTAL RESULTS WITH A SIMPLIFIED SYSTEM}

To demonstrate the feasibility of our design, we have performed experiments with a simplified version of our proposed system shown in Fig.3. Here, only Alice holds a phase modulator, while Bob always chooses the same measurement basis. Strictly speaking, no genuine secret key can be distributed by this simplified system, but it allows us to evaluate the performance of a fully developed system (as shown in Fig.2) in terms of stability and quantum bit error rate (QBER). In Fig.3, the cw output from a $1550 \mathrm{~nm}$ laser (L) is modulated by an amplitude modulator (AM) to generate 500ps laser pulses. Each laser pulse is split into $S_{1}$ and $S_{2}$ at a symmetric fiber coupler, which go through a long fiber loop $(L 1+L 2 \approx 40 \mathrm{~km})$ in the clockwise and counterclockwise directions, respectively. The interference patterns at $\mathrm{Ch} 1$ and $\mathrm{Ch} 2$ are measured by two InGaAs single photon detectors (SPD, Id Quantique, id200), which work in gated mode. For a 5ns gating window, the overall detection efficiency is $\sim 10 \%$ and the dark count probability is $5 \times 10^{-5}$ per gating window. A fiber-pigtailed AOM (Brimrose inc.) is placed inside the fiber loop asymmetrically $(L 1-L 2 \approx 700 m)$. Due to this asymmetry, phase modulation between $S_{1}$ and $S_{2}$ can be achieved by modulating AOM's driving frequency, similar to the phase modulator shown in Fig.1a. Because of the birefringence in the fiber loop, the polarization states of $S_{1}$ and $S_{2}$ could be different after they go through the fiber loop [23]. This is compensated by a polarization controller (PC). The synchronization is achieved as follows: A pulse generator (PG), which is triggered by a function generator (FG1), drives the amplitude modulator (AM) to produce 500ps laser pulses. FG1 also triggers a delay generator (DG, Stanford research system, DG535), which in turn produces two gating signals for SPD1 and SPD2, and one trigger signal for a data acquisition card (NI, PCI-6115). The AOM is driven by another function generator FG2, whose frequency is controlled by the data acquisition card.

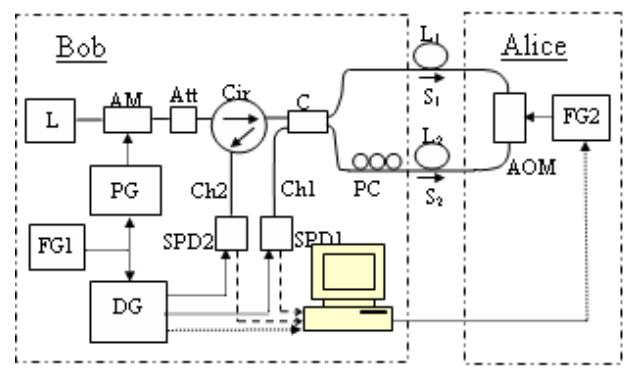

Fig. 3. Experimental setup: L-1550nm cw laser; AM-amplitude modulator; Att-attenuator; Cir-circulator; C-2x2 coupler; PC-polarization controller; FG1, FG2-function generator; PG-Pulse generator; DG-Delay generator; SPD1, SPD2-Single Photon detector

We measured the interference visibility by scanning the frequency of FG2 while recoding the outputs from the two SPDs. The average photon number per pulse (out from Alice's side) was set to be 0.8 , which matched with signal photon level in a decoy state QKD system [19-20]. The measured interference visibility for a $40 \mathrm{~km}$ and a $10 \mathrm{~km}$ fiber loop was $96 \%$ and $99 \%$ respectively.
To run the BB84 protocol, a random number file (1Kbits) is preloaded to the buffer of the data acquisition card. This random file contains a sequence of four discrete values corresponding to the four phase values in the BB84 protocol $\{0$, $\pi / 2, \pi, 3 \pi / 2\}$. Once triggered, the data acquisition card reads out a value from the random file and sends it to FG2 to encode Alice's phase information. The data acquisition card also samples the outputs from SPDs (Bob's measurement results) into its input buffer. In this preliminary setup, Bob always uses the same basis for his measurement. After transmitting $100 \mathrm{~K}$ bits, Alice and Bob can estimate the QBER by comparing the data contained in Alice's random file and Bob's measurement results. We ran the system continuously for one hour without any adjustment, the QBER drifted slowly from $2 \%$ to $5 \%$, as shown in Fig.4. In practice, to improve the long-term stability, simple recalibration process can be employed, as in other QKD systems. During this experiment, the pulse repetition rate was set to $1 \mathrm{KHz}$ for easy synchronization.

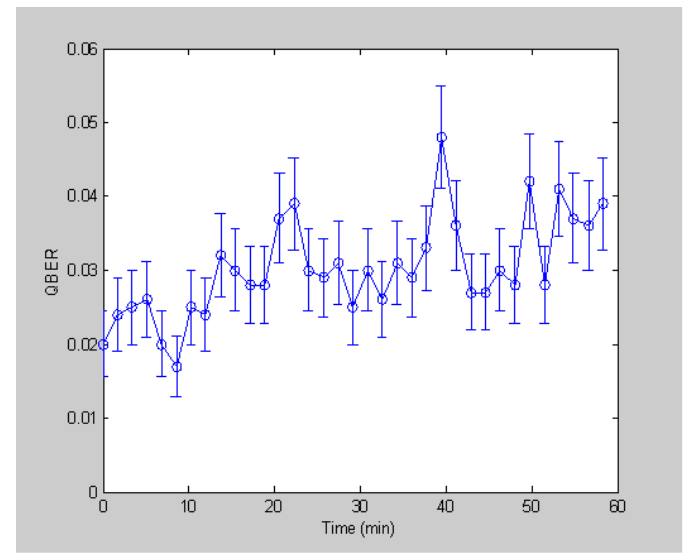

Fig. 4. Measured QBER in one hour without any feedback control. The photon level is 0.8 photon/pulse (The error bars indicate the statistic fluctuation due to the finite detection events)

For a typical AOM, its frequency modulation rate is in the range of $1 \sim 10 \mathrm{MHz}$, which is compatible with the operation rate of today's QKD system. The ultimate operating rate of our phase modulation scheme was tested by running FG2 in Frequency-Shift Keying mode: its frequency hopped between two values (corresponds to 0 or $\pi$ phase delay) at $100 \mathrm{KHz}$ rate. Note in this case, the equivalent phase-encoding rate was $200 \mathrm{KHz}$. Here, strong, cw laser was input to the system, and interference signal from $\mathrm{Ch} 1$ was detected with a photo detector. Fig.5 shows the experimental result, where the sharp rising edge $(\sim 500 \mathrm{~ns})$ indicates a potential phase modulation rate of a few $\mathrm{MHz}$ could be achievable.

\section{Discussion AND CONCLUSION}

In a bidirectional QKD system, such as the "plug \& play" system as well as the system we propose here, Alice allows signals to go in and out of her device, this opens a potential backdoor for Eve to launch various Trojan horse attacks. In our simplified setup, where only one AOM is used in Alice's side as a phase modulator, the frequency of the laser pulse 


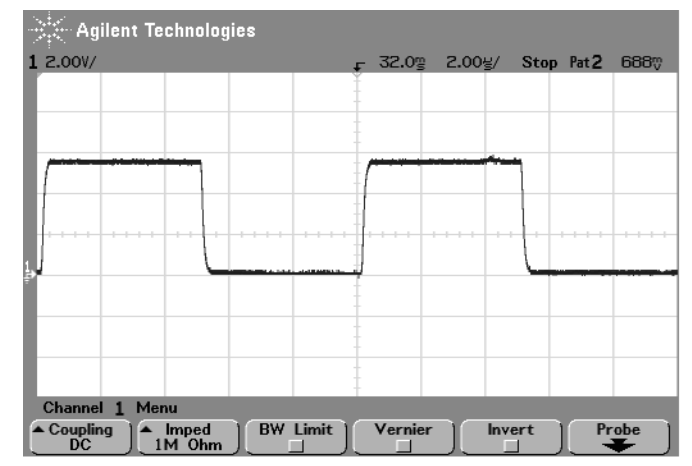

Fig. 5. The experimental results at $200 \mathrm{KHz}$ Phase modulation rate. The sharp rising edge $(\sim 500 \mathrm{~ns})$ indicates a potential phase modulation rate of a few $\mathrm{MHz}$ could be achievable.

output from Alice depends on the encoded phase information (due to the frequency shift induced by the AOM). This may leak additional information to Eve and a naive application of the unconditionally secure proof of BB84 to our current experimental set-up is invalid.

In the standard BB84 protocol, Alice encodes phase information $\{0, \pi / 2, \pi, 3 \pi / 2\}$ by modulating the $\mathrm{AOM}$ at frequency $\left\{f_{0}, f_{0}+\Delta f, f_{0}+2 \Delta f, f_{0}+3 \Delta f\right\}$. From (2), the encoded phase depends on both the AOM's driving frequency $f$ and the fiber length difference $\Delta L$. In principle, Eve can build up a device with different fiber length, breaks into the communication channel, and plays the "intercept and resend" attack. Suppose Eve uses her device to send laser pulses to Alice. Unaware that the pulses are from Eve, Alice will still shift the frequency of light by one of the values $\left\{f_{0}, f_{0}+\Delta f\right.$, $\left.f_{0}+2 \Delta f, f_{0}+3 \Delta f\right\}$. By choosing a suitable fiber length difference, Eve can re-mapping the encoded phase from $\{0$, $\pi / 2, \pi, 3 \pi / 2\}$ to $\{0, \delta, 2 \delta, 3 \delta\}$, where $\delta$ is under Eve's control. In [24], we describe detail of this "phase-remapping attack" and proved that Eve can learn the full information of the final key at the cost of introducing a $14.6 \%$ QBER. Note that this number is substantially lower than the proved secure bound of $18.9 \%$ for the standard BB84 protocol [25]. We remark that this loophole can be closed by employing the phase modulator shown in Fig.1b, which introduces no frequency shift (as the setup in Fig.2).

In conclusion, we propose a polarization-insensitive phase modulation scheme and a stable Sagnac QKD system employing this technique. Compared with previous Sagnac QKD schemes based on polarization-sensitive phase modulators, our proposed system demonstrated better performance over longer fiber. Preliminary experimental results showed an interference visibility of $96 \%(99 \%)$ for a $40 \mathrm{~km}(10 \mathrm{~km})$ fiber loop at single photon level. With this novel polarization-insensitive phase modulation scheme, we expect the performance of many practical QKD systems can be greatly improved.

\section{ACKNOWLEDGMENT}

Financial supports from NSERC, CRC Program, CFI, OIT, PREA, and CIPI are gratefully acknowledged.

\section{REFERENCES}

[1] C. H. Bennett and G. Brassard, in Proc. of IEEE Int. Conf. on Computers, Systems, and Signal Processing, IEEE, 175-179 (1984).

[2] N. Gisin, G. Ribordy, W. Tittel, and H. Zbinden, Rev. Mod. Phys. 74, 145-195 (2002).

[3] D. Mayers, Unconditional security in quantum cryptography, J. of ACM 48 (2001), 351-406, Preliminary version in Mayers, D. Advances in Crytology-Proc. Crypto '96, vol. 1109 of Lecture Notes in Computer Science, Koblitz, N. Ed. (Springer-Verlag, New York, 1996), pp. 343357.

[4] H. -K. Lo and H. F. Chau, Science, 283, 2050-2056 (1999).

[5] P. W. Shor and J. Preskill, Phys. Rev. Lett. 85 (2000), 441.

[6] D. Gottesman, H.-K. Lo, N. Ltkenhaus, and J. Preskill,D. Gottesman, Quantum Information and Computation 4, 325-360 (2004).

[7] R. J. Hughes, G. L. Morgan, and C. G. Peterson, J. of Mod. Opt., 47, 533-547 (2000)

[8] Z. L. Yuan and A. J. Shields, Opt. Express, 13, 660-665 (2005).

[9] A. Muller, T. Herzog, B. Huttner, W. Tittel, H. Zbinden, and N. Gisin Appl. Phys. Lett. 70, 793-795 (1997).

[10] T. Nishioka, H. Ishizuka, T. Hasegawa, and J. Abe, IEEE Photonics Technol. Lett., 14, 576-578 (2002).

[11] C. Y. Zhou and H. P. Zeng, Appl. Phys. Lett. 82, 832-834 ( 2003).

[12] C. Y. Zhou, G. Wu, L. E. Ding and H. P. Zeng, Appl. Phys. Lett. 83, 15-17 ( 2003).

[13] P. D. Kumavor, A. C. Beal, S. Yelin, E. Donkor, B. C. Wang, J. Lightwave Technol. 23, 268-276 (2005).

[14] W. Y. Hwang, Phys. Rev. Lett. 91, 057901 (2003).

[15] H.-K. Lo, in Proceedings of 2004 IEEE ISIT (IEEE Press, New York, 2004), p. 137, and presentation at the Fields Institute Conference on QIQC, July, 2004. http://www.fields.utoronto.ca/programs/scientific/04-05/quantumIC/abstracts/lo.ppt

[16] H.-K. Lo, X. Ma, and K. Chen, Phys. Rev. Lett. 94, 230504 (2005)

[17] X. B. Wang, Phys. Rev. Lett. 94, 230503 (2005).

[18] X. B. Wang, Phys. Rev. A 72012322 (2005).

[19] X. Ma, B. Qi,Y. Zhao, and H.-K. Lo, Phys. Rev. A 72, 012326 (2005).

[20] Y.Zhao, B. Qi, X. Ma, H.-K. Lo, and L. Qian, Preprint quant-ph/0503192 at http://arxiv.org (2005).

[21] F. Grosshans, G. V Assche, J. Wenger, R. Brouri, N. J. Cerf, and P. Grangier, Nature, 421, 238-241 (2003)

[22] A. Stefanov, H. Zbinden, N. Gisin, A. Suarez, Phys. Rev. A. 67, 042115 (2003).

[23] D. B. Mortimore, J. Lightwave Technol., 6, 1217-1224 ( 1988).

[24] C.-H.F. Fung, B. Qi, K. Tamaki, H.-K. Lo, "Phase-Remapping Attack in Quantum Key Distribution Systems", submit to ISIT 2006

[25] D. Gottesman and H.-K. Lo, IEEE Trans. Inform. Theory 49, 457 (2003). 